\title{
利用偶极子模型研究肌球蛋白 VI的定向运动
}

\author{
(1) 河北工业大学生物物理研究所, 天津 300401; \\ (2) 甘部学院物理系, 甘期 056005 \\ E-mail:1cpedu@yahoo.com.cn
}

李晨璞 ${ }^{\circledR}$, 韩英荣 $^{\circledR}$, 展永 ${ }^{\circledR}$, 张秀欣 $^{\circledR}$, 郑燕 ${ }^{(2)}$

2007-09-05 收稿, 2008-02-17 接受

国家自然科学基金(批准号：10645006)、高等学校博士学科点科研专项基金(编号：20060080001)及河北省自然科学基金(批准号: C2005000011)资助项目

摘要 从整个肌球蛋白分子马达系统的结构特点和实验现象出发, 利用电偶极子模型构 建一个势能函数, 应用朗之万方程讨论其定向运动行为. 通过调整分子马达电偶极子参 数, 模拟得到了肌球蛋白 VI分子马达系综沿微丝负向的平均位移和平均粒子流, 并讨论 了负载力和马达偶极子转动速率对肌球蛋白 VI系综运动的影响. 研究发现, 分子马达的 运动方向会随马达偶极子旋转方向的不同而变化, 马达偶极子逆时针旋转时分子马达向

关键词

分子马达

肌球蛋白 VI

朗之万方程

电偶极子 微丝负端运动(此时对应肌球蛋白 VI), 顺时针旋转时分子马达向正端运动(此时对应肌球 蛋白 V); 当负载力很大时, 肌球蛋白 VI甚至会向微丝的正端运动.

分子马达(molecular motor)是一类蛋白质, 它们 既具有酶的活性, 又具有运动活性, 通过催化ATP水 解, 将ATP分子水解释放的化学能转化成机械能而产 生定向运动. 细胞内部存在着广泛的物质运输, 同时 细胞间也不断地进行着物质交换, 分子马达在这些 过程中发挥着重要作用. 分子马达的种类很多, 从它 们的运动方式来看, 有旋转分子马达和线性分子马 达. 旋转分子马达中最具有代表性的是 ATP合成酶, 而线性分子马达主要包括肌球蛋白(myosin)、驱动蛋 白(kinesin)和动力蛋白(dynein)等. 分子马达有高效 的能量转化机制和定向运动的性质. 大部分分子马 达的能量转化效率都在 $60 \%$ 以上, ATP合成酶甚至能 达到 $100 \%$, 因此越来越多的研究人员开始关注分 子马达, 并投入到分子马达的研究中. 细胞中分子马 达种类繁多, 并且胞内环境复杂多样, 但是各种分子 马达却能有条不紊地沿着各自的轨道运动并执行其 功能.

虽然肌球蛋白 VI (myosin VI) 相对其他分子马达 来说被研究得较晚, 但它在细胞内的多种功能却引 起了人们的关注, 其中最值得注意的就是其约 $36 \mathrm{~nm}$ 的步长和向着微丝负端的运动. 由于苂光标记和光
钳等实验技术的发展, 使得人们从微观上认识分子 马达成为可能. 基于肌球蛋白 VI的大量实验现象和 数据的积累, 研究人员对其提出了多种运动模型, 如 “热位点(hot spot)”模型 [2]、带有方向性的定长杆臂旋 转模型 [3]、带有可变区域(flexible domain)的模型 ${ }^{[4]}$ 、 交臂(hand-over-hand)扩散模型 [5,6]等. 人们通过不 同的模型去解释肌球蛋白 VI的大的步长, 其中交 臂模型通过电荷机制还解释了肌球蛋白 VI 的反向 运动.

实验现象的发现要求理论上的解释. 目前研究 分子马达的理论计算方法主要有两种, 一种是把分 子马达看成是在非对称周期势中运动的布朗粒子, ATP的水解过程看作非平衡涨落, 从而来研究分子马 达的动力学行为, 描述其动力学的方程通常称为朗 之万方程(Langevin equation) $)^{[7,8]}$. 另一种是把分子马 达运动的一个周期看成几个分离的态, 分子马达在 各个态之间进行跃迁, 描述分子马达运动的方程称 作主方程(Master equation) $)^{[9,10]}$. 本文主要利用朗之万 方程通过简单的电偶极子模型对肌球蛋白 VI进行研 究. 在此模型中, 肌球蛋白 VI可以产生步长约 $36 \mathrm{~nm}$ 沿微丝负向的运动, 并且发现分子马达电偶极子的 
旋转方向可改变分子马达在微丝上的运动方向, 而 分子马达的运动速度也会随负载力的增加而减小, 如果负载力足够大则分子马达会产生反向的运动, 所得结果与实验现象定性地吻合.

\section{1 生物基础}

肌球蛋白是以肌动蛋白微丝为运动轨道的线性 分子马达. 肌球蛋白是一个大家族, 目前根据其蛋白 氨基酸序列的不同分成 18 个种类. 其中关于肌球蛋白 $\mathrm{V}($ myosin $V)$ 和肌球蛋白 $V I$ 的研究较多, 因为它们在 胞内发挥重要的作用, 如囊泡的运输、微线毛的形成 和细胞的分裂 $[11$ 等.

肌球蛋白 $\mathrm{V}, \mathrm{VI}$ 二聚体是由两个对等的单体组 成, 每个单体包括一头部区域、轻链区域(IQ模体)、 卷曲螺旋域和一球形尾域 [12,13]. 在头部区域有两个 位点, 一个位点和微丝连接称为连接位点, 另一位点 催化核苷酸ATP水解称为催化位点. 卷曲螺旋域被认 为是单体二聚化的区域, 而球形区域则连接被运输 的物质. 它们的不同之处在于, 肌球蛋白 $\mathrm{V}$ 头部有一 个插入体, 颈部有六个IQ模体, 它向微丝正端运动. 而肌球蛋白 VI头部有两个插入体，颈部只有一个IQ 模体，由于第二个插入体的存在，使得肌球蛋白 VI向 微丝负端运动 $[14,15]$.

微丝是肌球蛋白运动的轨道, 是由肌动蛋白单 体聚合成的有极性的双螺旋链, 这些单体也是有极 性的, 由于单体的极性最终导致了整个微丝的极性. 大部分微丝的长度处在动态的变化中, 正极生长快, 负极生长慢, 即“踏车”现象. 由于肌动蛋白单体极性 的存在, 所以在文献 [16]的模型中, 微丝被看成多个 偶极子连成的双螺旋链.

肌球蛋白 $V, V I$ 分子马达头部有两个有连接功能 的位点, 其中连接位点附近主要是正电荷, 而连接核 甘的催化位点周围主要存在的是负电荷 $[16]$, 因此分 子马达的两头部被近似看作两个电偶极子. 在分子 马达沿微丝运动过程中, ATP的连接、水解和产物释 放使分子马达头部的构象发生变化, 这样就引起了 头部电荷分布的空间变化, 即分子马达的运动过程 对应着马达头部偶极子的旋转. 另外两头部上ATP的 连接、水解, 与产物的释放过程是不同步的 ${ }^{[171}$, 于是 对于分子马达整体而言也可看成一个大的电偶极子, 此电偶极子的旋转被视作是分子马达的一内部自由 度 ${ }^{[18,19]}$.

\section{2 模型的建立}

对于运行在微丝上的肌球蛋白 VI, 它的步长大 约是 $36 \mathrm{~nm}$, 此长度正好是微丝半周期的长度. 在它 运行一步的过程(图 $\left.1^{[5]}\right)$ 中, 首先分子马达L头部和微 丝上 $\mathrm{A}$ 单体连接, 然后连接 $\mathrm{L}$ 头部的杆臂做一个功冲 击 (powerstroke), 把后边的 $\mathrm{T}$ 头部甩向前边. 当做功 冲击结束时, 和 $\mathrm{T}$ 头部最接近的微丝单体就是距 $\mathrm{L}$ 头 部长约为 $36 \mathrm{~nm}$ 的B单体. 由于在溶液中粒子间的电 相互作用力随距离的增加以负指数形式减小 [20]，而 在做功冲击过程中, $\mathrm{T}$ 头部距离 $\mathrm{A}, \mathrm{B}$ 单体中间的其他 单体相对很远, 因此这些单体对分子马达的作用力 可以忽略. 当把分子马达整体看成一个粒子时, 它在 运动中只受到像 $\mathrm{A}, \mathrm{B}$ 这样间隔大约为 $36 \mathrm{~nm}$ 单体的作 用力. 所以微丝的结构可以看成如图 2 所示的模型, 其中 $P_{1}$ 和 $P$ 分别代表微丝轨道电偶极子的偶极矩和分 子马达电偶极子的偶极矩.

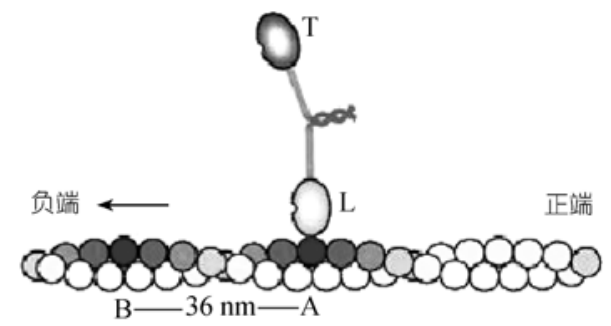

图 1 分子马达做功冲击

肌球蛋白 VI比肌球蛋白 $V$ 的颈部短很多, 但是 这并没有影响到肌球蛋白 $V \mid$ 和肌球蛋白 $V$ 相近的 36 $\mathrm{nm}$ 步长 ${ }^{[2.21]}$, 因为肌球蛋白 VI卷曲螺旋域的一部分 可以被打开 ${ }^{[4]}$, 在它的运动中起到延长杆臂的作用. 因此运动方向成为两者的主要差异, 由于肌球蛋白

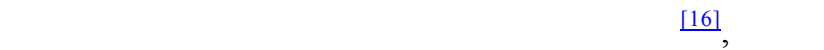
中用分子马达电偶极子的旋转方向来区别二者, 肌 球蛋白 VI的偶极子逆时针旋转, 而肌球蛋白 V 的偶 极子顺时针旋转.

取一段微丝, 建立如图 2 所示的直角坐标系, 得 到微丝周围的电势为

$$
V(x, y, z)=V_{0} \sum_{n=-100}^{100} \frac{x-n d}{\left[(x-n d)^{2}+\left(y-y_{n}\right)^{2}+\left(z-z_{n}\right)^{2}\right]^{3 / 2}},
$$

其中 $V_{0}=\frac{P_{1}}{4 \pi \varepsilon}, P_{1}$ 为微丝轨道电偶极子的偶极矩, $\varepsilon$ 为 蛋白质平均介电常数, $d$ 是微丝半周期的长度. 微丝 周围电势 $V(x, y, z)$ 的势曲线如图 3 所示. 


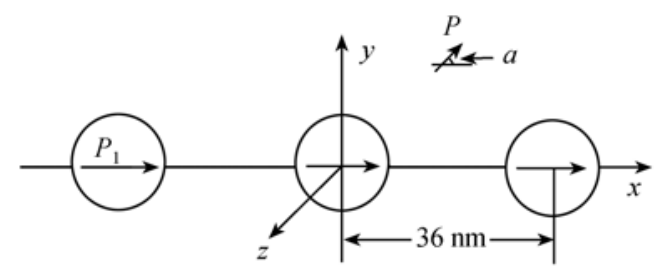

图 2 分子马达和微丝系统的偶极子模型

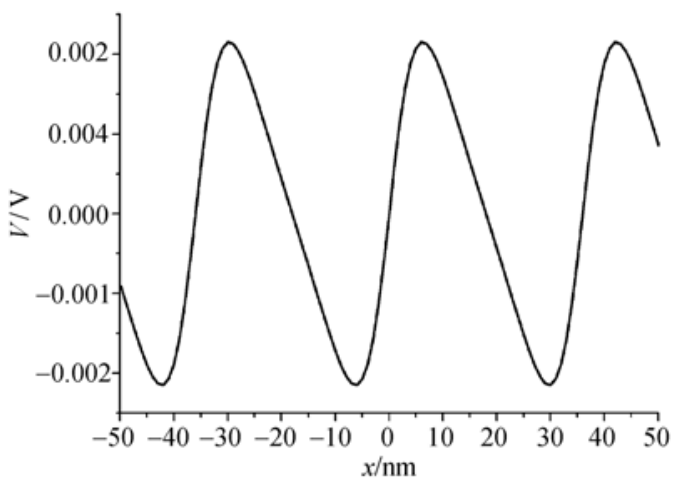

图 3 微丝周围的电势

\section{3 计算过程}

分子马达受到(1)式所示的周期电场的作用，因 ATP 的连接、水解和产物的释放过程对应分子马达偶 极子的旋转, 且设转动速率为 $\omega$, 所以分子马达偶极 子旋转的周期 $T$ 也就对应着核苷的周期变化. 由于分 子马达所处的溶液环境复杂, 不断受小分子的碰撞, 模型中把这些碰撞看成分子马达受到的外界白噪声. 这样分子马达就被看作是在周期电场中运动, 并不 断有非平衡涨落的布朗粒子. 于是分子马达的动力 学方程用朗之万方程表示为

$$
m \ddot{r}=f(r)-\gamma \dot{r}+\xi(t)+F,
$$

其中 $m$ 是分子马达的质量, $f(r)=-\partial W(r, t) / \partial r$, $W(r, t)$ 是分子马达偶极子处在微丝电势中任一点的 能量, $r$ 代表 $x, y, z, \gamma$ 是阻尼常数, $r$ 上的圆点表示对 时间 $t$ 求导数, $F$ 是分子马达所受的常负载力, $\xi(t)$ 是 随机力, 具有高斯白噪声的形式. $\xi(t)$ 满足

$$
\begin{gathered}
\langle\xi(t)\rangle=0, \\
\left\langle\xi(t) \xi\left(t^{\prime}\right)\right\rangle=2 B \delta\left(t-t^{\prime}\right),
\end{gathered}
$$

$B$ 是噪声强度, 与环境的温度有关.

只考虑分子马达偶极子在 $X-Y$ 平面内转动的情 况, 则微丝周围电势(1)式变成

$$
V(x, y)=V_{0} \sum_{n=-100}^{100} \frac{x-n d}{\left[(x-n d)^{2}+\left(y-y_{n}\right)^{2}\right]^{3 / 2}},
$$

而分子马达偶极子和微丝周围电场的相互作用能为

$$
W(x, y, t)=\vec{P} \cdot \nabla V,
$$

其中 $P$ 是分子马达偶极子的偶极矩, 且

$$
\begin{aligned}
\frac{\partial V}{\partial x} & =V_{0} \sum_{n=-100}^{100} \frac{R^{3}-3 R(x-n d)^{2}}{R^{6}}, \\
\frac{\partial V}{\partial y} & =V_{0} \sum_{n=-100}^{100} \frac{-3 R(x-n d)\left(y-y_{n}\right)}{R^{6}}, \\
R & =\sqrt{(x-n d)^{2}+\left(y-y_{n}\right)^{2}} .
\end{aligned}
$$

把(5)式代入(6)式，同时考虑(7)式，则可得到

$$
\begin{aligned}
W(x, y, t) & =P_{x} \frac{\partial V}{\partial x}+P_{y} \frac{\partial V}{\partial y} \\
= & P \cos \alpha \frac{\partial V}{\partial x}+P \cos \beta \frac{\partial V}{\partial y} \\
= & P \cos \left(\omega t+\alpha_{0}\right) \frac{\partial V}{\partial x}+P \sin \left(\omega t+\alpha_{0}\right) \frac{\partial V}{\partial y},
\end{aligned}
$$

$\alpha$ 和 $\beta$ 是分子马达偶极子与两坐标轴间的夹角.

由于分子马达所处的溶液环境是过阻尼的, 所 以(2)式中的惯性项可以省略, 且对时间进行重新标 度, 则(2)式成为

$$
x=f(x)+\xi(\tau)+F .
$$

对(9)式两边取平均, 则可以得到分子马达的平均速度

$$
\langle\dot{x}\rangle=\langle f(x)+F\rangle+\langle\xi(\tau)\rangle .
$$

把(3)式代入(10)式, 且考虑 $F$ 是一常量, 所以分子马 达的平均速度为

$$
\langle\dot{x}\rangle=\langle f(x)\rangle+F .
$$

同样，对(9)式通过运用 Runge-Kutta 二阶算法(也叫 预值修正算法)进行数值模拟, 可以得到分子马达位 移曲线.

\section{4 分析}

\section{1 平均位移 $\langle x\rangle$ 和粒子流 $\langle v\rangle$}

由于(9)式是一个随机方程, 一个分子马达的位 移和速度具有随机性, 所以我们计算由多个马达组 成的系综的粒子流和位移，其中系综内分子马达间 的相互作用被忽略. 图 4 和 5 是通过使分子马达偶极 子 $P$ 逆时针转动, 对分子马达系综进行数值模拟运算 而得到的肌球蛋白 VI的平均位移和粒子流随时间 $t$ 的变化曲线, 如果令分子马达偶极子 $P$ 顺时针转动, 则可得到肌球蛋白 $V$ 沿微丝正向的运动位移和粒子 流(未给出图示). 从图 4 和 5 可以看出: (1) 在平均位 移曲线上，肌球蛋白 VI随时间有稳定的梯跳运动，每 


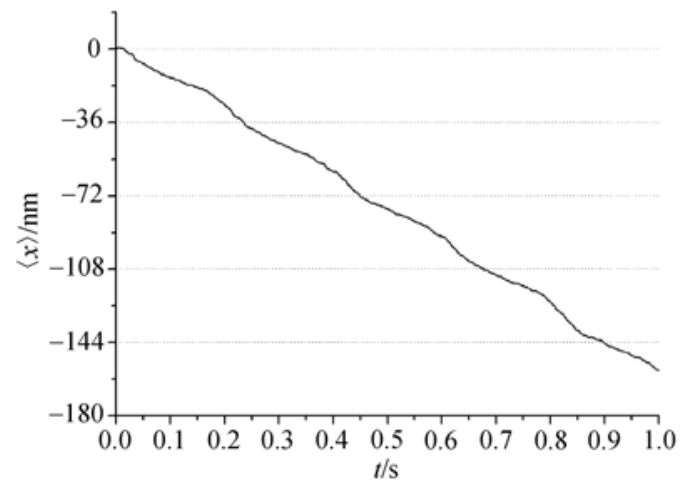

图 $4\langle x\rangle-t$ 曲线

$D=0.3, \omega=10 \pi, \Delta t=0.001 \mathrm{~s}, F=0$

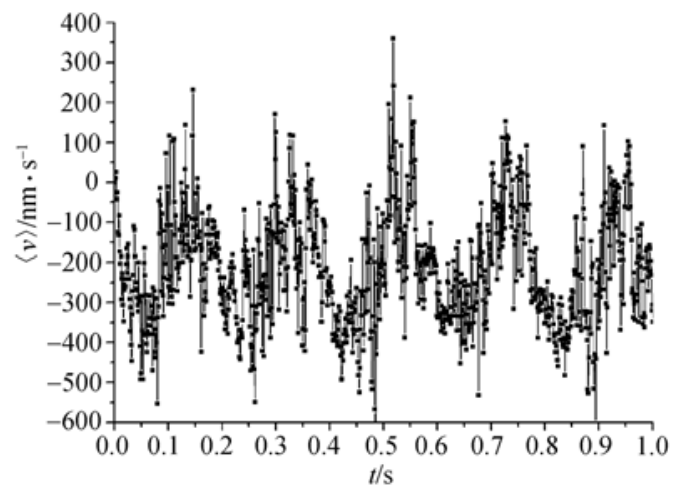

图 $5\langle v\rangle-t$ 曲线

$D=0.3, \omega=10 \pi, \Delta t=0.001 \mathrm{~s}, F=0$

次跳跃的步长约 $36 \mathrm{~nm}$, 这与微丝半周期长度相当. 肌球蛋白 VI进行定向运动, 并且是向微丝的负端运 动. (2) 在给定马达偶极子转动速率 $\omega=10 \pi$ 的情况 下，肌球蛋白 VI粒子流的数值在 $200 \mathrm{~nm} / \mathrm{s}$ 左右周期 波动, 并且波动周期与分子马达偶极子的转动周期 $T$ $=2 \pi / \omega=0.2 \mathrm{~s}$ 相一致.

4.2 分子马达偶极子的转动速率 $\omega$ 对平均粒子流 $\langle v\rangle_{T}$ 的影响

为了更清楚地认识肌球蛋白 VI马达偶极子转动 速率 $\omega$ 对肌球蛋白 VI动力学行为的影响, 我们对图 5 所示的粒子流进行时间平均, 这样可以得到肌球蛋 白 $\mathrm{VI}$ 的平均粒子流 $\langle v\rangle_{T}$. 平均粒子流 $\langle v\rangle_{T}$ 随马达偶极 子转动速率 $\omega$ 的变化曲线如图 6 所示.

由图可以看到当转动速率 $\omega$ 很小 $(\omega=0.1 \pi)$ 或者 是很大 $(\omega=1000 \pi)$ 时, 平均粒子流都很小但又略有差 别. 当 $\omega=0.1 \pi$ 时, 虽然马达偶极子转动得很慢, 但是 马达偶极子与微丝电势的相互作用仍然能使肌球蛋 白 VI越过能量势垒, 所以此时仍有很小的平均粒子 流. 而当 $\omega=1000 \pi$ 时, 由于马达偶极子转动得很快,

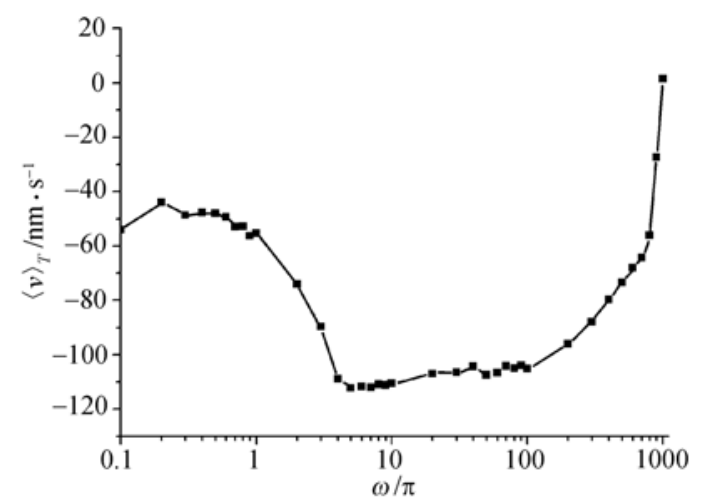

图 6 平均粒子流 $\langle v\rangle_{T}$ 随分子马达偶极子转动速率 $\omega$ 的 变化曲线

$D=0.3, \Delta t=0.001 \mathrm{~s}, F=0$

使得肌球蛋白 VI粒子的运动远滞后于分子马达与微 丝间相互作用势的变化, 此时平均粒子流的数值近 似为零. 当 $\omega$ 取 $0.1 \pi \sim 1000 \pi$ 数值时, 肌球蛋白 VI有明 显的平均粒子流. 可见肌球蛋白 VI对转动速率具有 选择性, 而不是简单的线性关系. 在电偶极子相互作 用模型中, 马达偶极子的转动对应分子马达头部区 域的核苷的变化, 既然肌球蛋白 VI对马达偶极子转 动速率的大小有选择性, 则马达头部区域连接核苷 的速度也不能太快或太慢, 而应该取一个合适的数 值来协调分子马达的运动.

\section{3 平均粒子流 $\langle v\rangle_{T}$ 随负载力 $\boldsymbol{F}$ 的变化}

肌球蛋白 VI的一个重要功能就是沿着微丝向其 负端运输胞内的一些物质, 所以它在运动过程中一 定要受到被运物质对它的负载力. 图 7 显示出肌球蛋 白 VI平均粒子流随负载力 $F$ 的变化, 负值表示分子马 达受到负载力的方向与它的运动方向相反, 正值表 示两者方向相同.

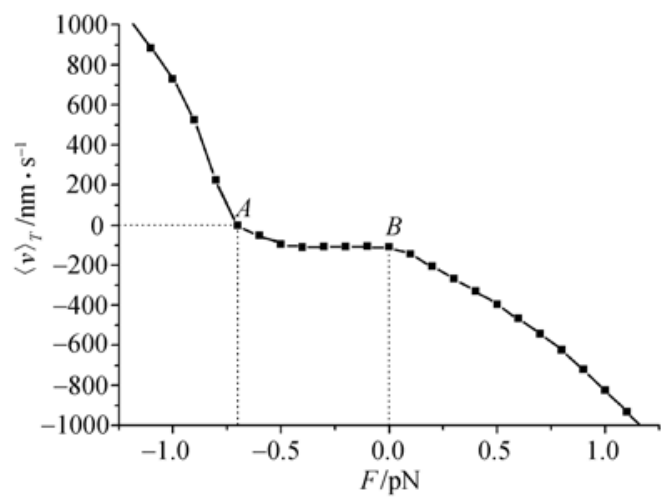

图 7 平均粒子流 $\langle v\rangle_{T}$ 随负载力 $F$ 的变化

$D=0.3, \omega=10 \pi, \Delta t=0.001 \mathrm{~s} ; A$ 点表示肌球蛋白 $\mathrm{Vl}$ 速度为零时的 值, $B$ 点是肌球蛋白 VI所受负载力为零时的点 
从图中可看出：(1) 当负载力和肌球蛋白 Vl运动 方向相同时(图 7 中 $B$ 点右方), 肌球蛋白 VI的平均粒子 流随负载力的增加而增大. (2) 当负载力和运动方向 相反时(图 7 中 $B$ 点左方), 肌球蛋白 VI的平均粒子流随 负载力的增大而减小, 并且当负载力的数值大于 0.7 $\mathrm{pN}$ (图 7 中 $A$ 点)时, 分子马达肌球蛋白 VI甚至会向微 丝的正端运动, 这一性质类似于肌球蛋白 $\mathrm{V}^{[22]}$.

\section{5 结论}

根据肌球蛋白 VI分子马达运动的特点和微丝的 结构特征, 建立了微丝和分子马达间相互作用的电 偶极子模型. 通过偶极子间的电相互作用，从理论上 对肌球蛋白 VI的运动情况做了定性讨论, 得到: (1) 通过调整马达偶极子转动方向可以使肌球蛋白 VI产 生指向微丝负端的定向运动. (2) 肌球蛋白 VI和微丝
构成的整个系统对马达偶极子的转动速率具有选择 性, 并不是简单的线性关系. (3) 肌球蛋白 VI的平均 粒子流随负载力的方向和大小而变化, 甚至在一定 条件下能产生反向粒子流.

目前, 一些从肌球蛋白 VI的微观结构出发来研究 其运动的模型正在被不断地提出，甚至发展到分子水 平, 如根据肌球蛋白 VI轻链的弹性能量出发对其步长 和速度的研究 ${ }^{[23]}$, 根据肌球蛋白 VI特殊的亚结构单 元对其反向运动的研究 ${ }^{[14,15]}$ 等. 本文利用电偶极子模 型从朗之万方程出发对肌球蛋白 VI沿微丝负向的运 动和其 $36 \mathrm{~nm}$ 的步长等一些性质给出了定性半定量的 解释, 但是由于模型大大简化了肌球蛋白 VI自身的内 部结构，使得自身结构对马达运动性质的影响很容易 被忽略, 这一点仍值得以后进一步讨论.

\section{参考文献}

1 Karplus M, Gao Y Q. Biomolecular motors: The F F $_{1}$ ATPase paradigm. Curr Opin Struct Biol, 2004, 14: 250-259 [doi]

2 Nishikawa S, Homma K, Komori Y, et al. Class VI myosin moves processive along actin filaments backward with large steps. Biochem Biophys Res Commun, 2002, 290(1): 311-317[doi]

3 Lister I, Schmitz S, Walker M, et al. A monomeric myosin VI with a large working stroke. EMBO J, 2004, 23: 1729-1738 [doi]

4 Rock R S, Ramamurthy B, Dunn A R, et al. A flexible domain is essential for the large step size and processivity of myosin VI. Mol Cell, 2005, 17: 603-609 [doi]

5 Xie P, Dou S X, Wang P Y. A hand-over-hand diffusing model for myosin-VI molecular motors. Biophys Chem, 2005, 122: 90-100[doi]

6 Xie P, Dou S X, Wang P Y. Model for processive movement of myosin V and myosin VI. Chin Phys, 2005, 14(4): 7440-752

7 Keller D, Bustamante C. The mechanochemistry of molecular motors. Biophys J, 2000, 78(2): 541-556

8 Zhan Y, Zhao T J, Yu H, et al. The steping motion of Brownian particle derived by nonequilibrium fluctuation. Commun Theor Phys, 2003, 39(6): 653-656

9 Kolomeisky A B, Fisher M E. A simple kinetic model describes the processivity of myosin-V. Biophys J, 2003, 84(3): 1642-1650

10 吴魏霞，赵同军，展永，等。分子马达随机跃迁暂态特征。河北工业大学学报, 2003, 32(2): 15-20

11 Frank D J, Noguchi T, Miller K G. Myosin VI: A structural role in actin organization important for protein and organelle localization and trafficking. Curr Opin Cell Biol, 2004, 16: 189-194[doi]

12 Bahloul A, Chevreux G, Wells A L, et al. The unique insert in myosin VI is a structural calclum-calmodulin binding site. Proc Natl Acad Sci USA, 2004, 101(14): 4787-4792[doi]

13 Hasson T, Mooseker E M. Porcine myosin-VI: Characterization of a new mammalian unconventional myosin. J Cell Biol, 1994, 127: 425440 [doi]

14 Ménétrey J, Bahloul A, Wells A L, et al. The structure of the myosin VI motor reveals the mechanism of directionality reversal. Nature, 2005, 435: 779-785[ [doi]

15 Park H, Li A, Chen L Q, et al. The unique insert at the end of the myosin VI motor is the sole determinant of directionality. Proc Natl Acad Sci USA, 2007, 104(3): 778-783[doi]

16 Masuda T. An electromechnical model of myosin molecular motor. J Theor Biol, 2003, 225: 507-515 [doi]

17 De La Cruz E M, Ostap E M, Sweeney H L. Kinetic mechanism and regulation of myosin VI. J Biol Chem, 2001, 276(34): 32373-32381 [doi]

$18 \mathrm{Yu} \mathrm{H}$, Zhao T J, Ji Q, et al. Dipole-dipole interaction and the directional motion of Brownian motors. Commun Theor Phys, 2002, 37(3): 381-384

19 Zhang H W, Zhi L H, Li Y X, et al. Brownian motors based on electric dipole interaction. Modern Phys Lett B, 2003, 17(7): 281-289[doi]

20 Levin Y. Electrostatic correlations: From plasma to biology. Rep Prog Phys, 2002, 65: 1577-1632[doi]

21 Rock R S, Rice S R, Wells A L, et al. Myosin VI is a processive motor with a large step size. Proc Natl Acad Sci USA, 2001, 98(24): $13655-13659$ [doi]

22 Gebhardt J C M, Clemen A E-M, Jaud J, et al. Myosin-V is a mechanical ratchet. Proc Natl Acad Sci USA, 2006, 103(23): 8680-8685[doi]

23 Lan G H, Sun S X. Flexible light-chain and helical structure of F-actin explain the movement and step size of myosin-VI. Biophys J, 2006, 91: 4002-4013[ [doi] 